\title{
Advanced Motion Control: An Industrial Perspective
}

\author{
DCT 2003.15 \\ M. Steinbuch and M.L. Norg
}

European Journal of Control (1998) 278-293 and 294-297

1998 EUCA 


\title{
Advanced Motion Control: An Industrial Perspective*
}

\author{
M. Steinbuch and M. L. Norg \\ Philips Centre for Manufacturing Technology, Eindhoven, The Netheriands
}

Industrial feedback motion controllers are tuned using manual loopshaping, according to certain design rules. This controller tuning serves as a benchmark for robust control research as applied to optical and magnetic disk drive storage systems. The design and implementation of robust controllers for the focus servo of a compact disk and the tracking servo of a hard disk mechanism is investigated. The design objective is to achieve good trackfollowing performance in the presence of disk disturbances. $\mathrm{H}_{\infty}$ robust control is used to systematically include disturbance and specification models in the standard plant framework. The control design iteration is based on disturbance identification, including implementation within the design cycle. The industrial relevance of robust control is discussed and areas for academic research in robust control are indicated, as feedback from industry.

Keywords: Application; Compact disk; Disturbance rejection; Drives; Hard disk; Model-based control; Motion control; Robust control

\section{Introduction}

Model (or optimisation)-based control, in particular $\mathrm{H}_{\infty}$ and $\mathrm{H}_{2}$ control and its mixed forms, has seemingly matured as methodology to design highperformance control systems. One important unifying development has been the introduction of stan-

*The first version of this paper was presented at the European Control Conference, Brussels, 1-4 July 1997.

Correspondence and offprint requests to: $\mathrm{M}$. Steinbuch, Philips Centre for Manufacturing Technology, PO Box 218, $5600 \mathrm{MD}$ Eindhoven, The Netherlands. Email: m.steinbuch@philips.com dard plants ${ }^{1}$ as a means to systematically specify control design problems (see also Fig. 1).

The main task in translating a practical control design problem into this general format is the modelling issue: the nominal plant $P$, the disturbances $W_{d}$ acting on the system, the performance requirements $W_{p}$, and uncertainties $\Delta$ of the plant and possibly also of disturbances and specifications. The availability of various software packages and hardware tools then enables the control engineer to calculate the optimal controller $K$ and implement it. Hence, we can conclude: theory is ready and finished, and the industry is ready to start using it. Or not....

In this paper the starting point will be a general description of LTI SISO ${ }^{2}$ motion systems and their standard controller (Section 2), serving as a benchmark for our excursion into robust control of information storage systems - in particular, robust control

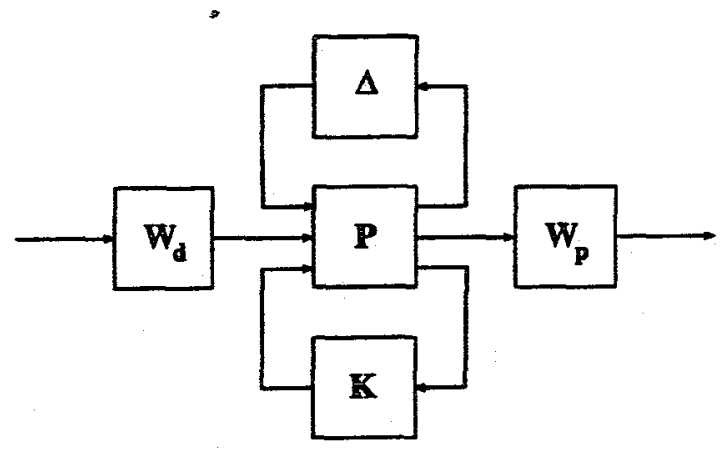

Fig. 1. Standard plant.

\footnotetext{
'Also called intelligent control paradigm, requiring definitions of inputs and outputs being a special case of the even more general viewpoint of behaviours [37].

2 Linear time invariant single-input single-output.

Received September 1997; Accepted in revised form August 1998 Recommended by M. Tomizuka and I. D. Landau
} 
of a compact disk drive (Section 3) and of a hard disk drive (Section 4), both illustrating a disturbance-based control design cycle. Based on the confrontation of robust control with these two applications, we will discuss the relevance of robust control for industry and emphasise areas for future research in robust control as seen from an industrial perspective (Section 5).

\section{Motion Control}

The application field of motion control systems is widely present in industry for motion tasks such as pick-and-place and robotics. The underlying disciplines as mechanics, electronic power supplies and electronics, electric motors, sensor technology and digital control have rapidly developed in the last decade. The field of mechatronic systems has emerged to emphasise the importance of a systems approach for design.

In this section we will describe ony a small but basic part of motion systems, namely the elementary positioning control loop. Consider a typical motion system as it is schematically depicted in Fig. 2.

The driving force $F$ is assumed to be generated by a current-controlled motor, such that the input of the system indeed can be seen as force. Either the motor position $x_{m}$ or the load position $x_{l}$ can be measured (in most cases unfortunately not both). Both masses (or inertias for rotating machines) are assumed to be connected via a mechanical connection with finite stiffness and damping. From the equations of motion, a simple linear dynamic model can be derived. The model has a frequency response as depicted in Fig. 3, both for the motor position measurement case $\left(F \rightarrow x_{m}\right)$ and for the load position measurement situation $\left(F \rightarrow x_{l}\right)$. The frequency responses are elementary for many motion control systems seen in practice $[31,36]$.

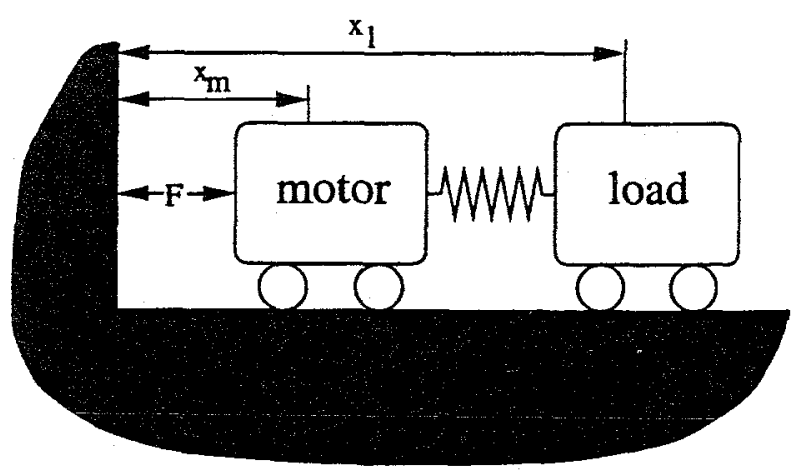

Fig. 2. Motion system.
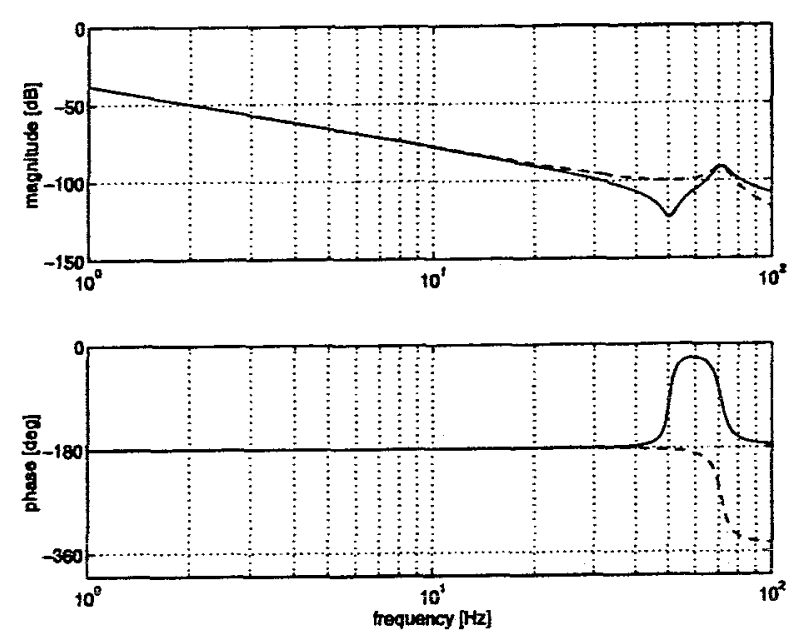

Fig. 3. Bode diagram of motion system, sensor on motor $(-)$ or load $(-)$.

A block diagram of the control loop is shown in Fig. 4. The use of acceleration feedforward is a standard means of obtaining small errors in case of tracking a setpoint signal. Such a feedforward signal is normally tuned on the basis of the assumption that the system can be approximately modelled as a single mass (i.e. a double integrator). In a more general format this would imply a two degree-offreedom control system. In this paper we will focus on the feedback loop only, since this is the situation for the CD and HDD control problems as described in the next sections.

The (feedback) controller for such a motion control system consists of the necessary stabilising PD part, in many cases in the form of a lead-lag filter $\left(\tau_{1} s+1\right) /\left(\tau_{2} s+1\right)$. In most cases integral action is added, as well as a low-pass filter at high frequencies to prevent amplification of noise, and possibly some notches to cope with parasitic dynamics.

Rules-of-thumb used in practice to tune a motion controller are given in terms of break-points of the various filters. If we denote the bandwidth, defined as the first $0 \mathrm{~dB}$ crossing of the open-loop frequency response, as $\omega_{b}$, then the integral action works until $\omega_{b} / 6$, the zero of the lead/lag is at $\omega_{b} / 3$, the pole is

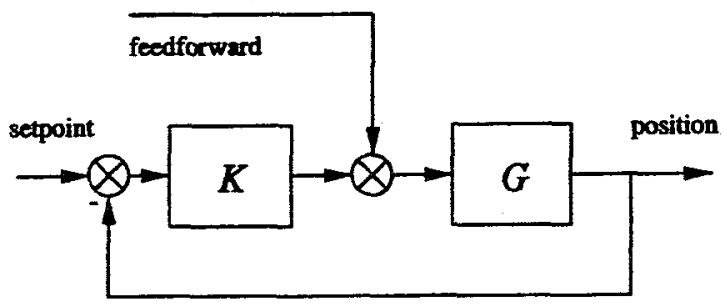

Fig. 4. Block diagram of a motion control system. 

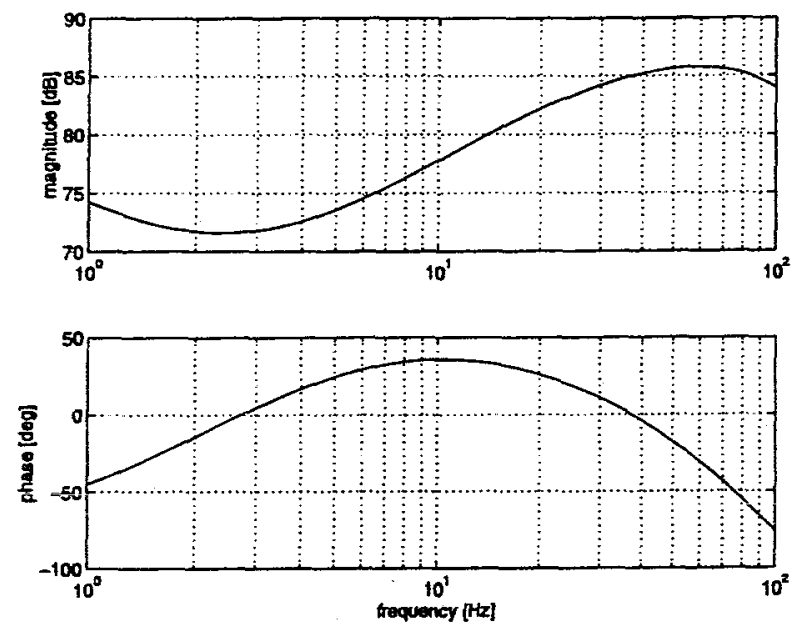

Fig. 5. Bode diagram of standard industrial motion controller.

at $\omega_{b} * 3$ and the low-pass starts at $\omega_{b} * 10$. A typical Bode diagram of a motion controller is shown in Fig. 5.

If stability is endangered by resonances, secondorder filters (generally called notches if the undamped frequency of the zeros equals that of the poles) are used to either obtain amplitude margin if one is certain about the damping of the resonances, or to change phase of the loop to have an amplitude robust solution. Playing with such filters and tuning while inspecting the open-loop frequency response (in Bode, Nyquist or Nichols charts) is what we call (manual) 'loopshaping'.

For the system above the controller gives the open-loop and closed-loop responses as depicted in Figs 6 and 7 . Of course, many interesting thoughts can be written down about the simple yet instructive
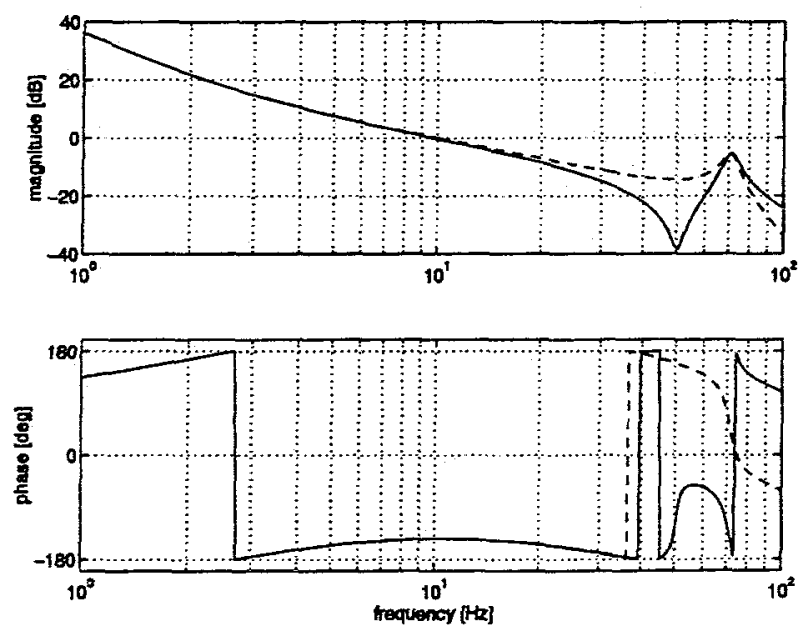

Fig. 6. Open-loop frequency response $G K(\omega)$ of controlled motion system, sensor on motor $(-)$ and on load $(-)$.
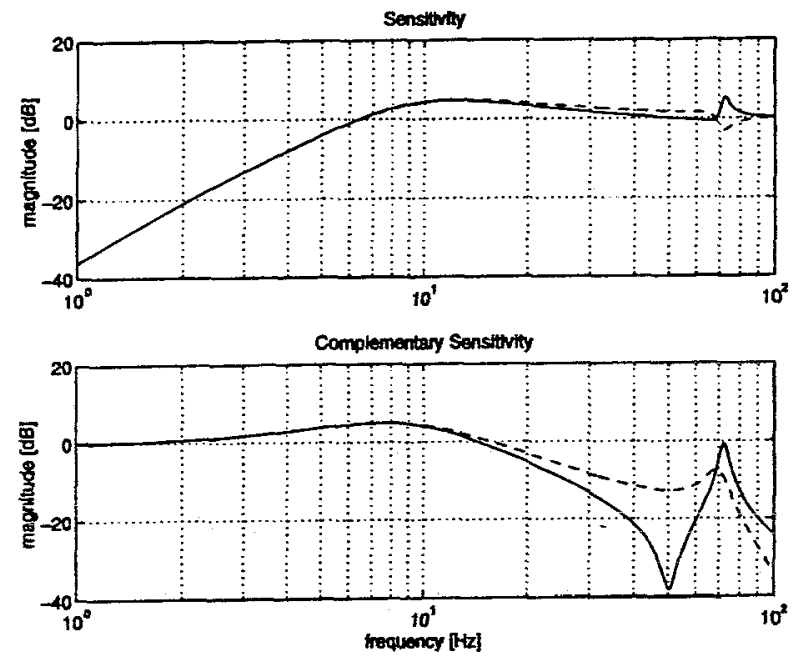

Fig. 7. Closed-loop frequency responses $(S=1 /(1+G K)$ and $T=G K S)$ of controlled motion system, sensor on motor $(-)$ and on load $(-)$.

example. One such thing is the robustness for the resonance in the load-controlled case (dashed line in Fig. 6): at the resonance frequency (i.e. at about $70 \mathrm{~Hz}$ ) the phase of the open-loop is close to zero degrees (hence in the right-half plane of the Nyquist diagram). This means that the closed-loop system is very robust for damping variations of the resonance. So, in this particular case it would be a waste to use a notch filter, in order to improve stability robustness for damping variations of the resonance. The phase for the co-located (motor) control case (solid line in Fig. 6) is much worse with respect to this robustness.

The controller tuning using loopshaping is the starting point for our investigation into storage drives, and serves, as a benchmark for performance assessment.

\section{Optical Disk Drive Control}

\subsection{Servo Control Problem}

An important research topic for optical data storage systems such as CD-ROM and its successor DVDROM is the possibility to increase the rotational frequency of the disk to obtain faster data readout and shorter access time [28]. In this section we will show the importance of control design for performance improvement of optical storage drives.

In Fig. 8 a schematic view of a compact disk (single-stage) mechanism is shown. The mechanism is composed of a turn-table DC motor for the rotation of the compact disk, and a balanced radial 


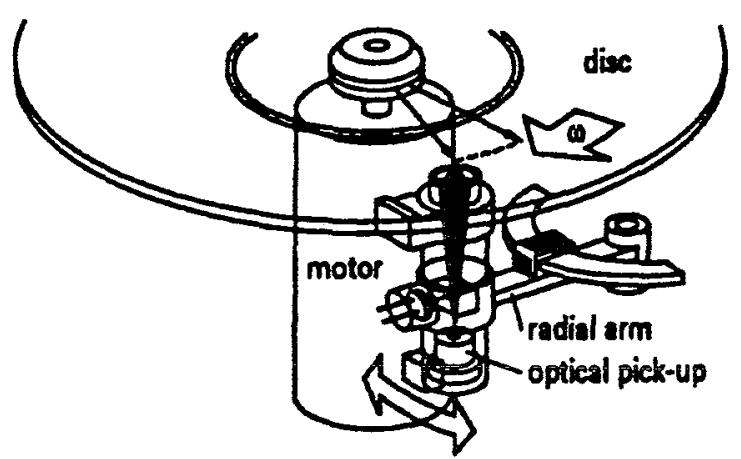

Fig. 8. Schematic view of a rotating arm compact disk mechanism.

arm for track-following. An optical element is mounted at the end of the radial arm. A diode located in this element generates a laser beam that passes through a series of optical lenses to give a spot on the information layer of the disk. An objective lens, suspended by two parallel leaf springs, can move in a vertical direction to give a focusing action. Although drives nowadays are using linear two-stage mechanisms with a long stroke sledge and a short stroke second actuator, the control design problem for the drive under investigation has equivalent properties.

Both radial and vertical (focus) position of the laser spot, relative to the track of the disk, have to be controlled actively. To accomplish this, the controller uses position-error information provided by four photo-diodes. As input to the system the controller generates control currents to the radial and focus actuator, which both are permanentmagnet/coil systems (VCM - voice coil motor).

In Fig. 9 a block diagram of the control system is shown. The radial and vertical track position are shown as output disturbance in Fig. 9. The relative

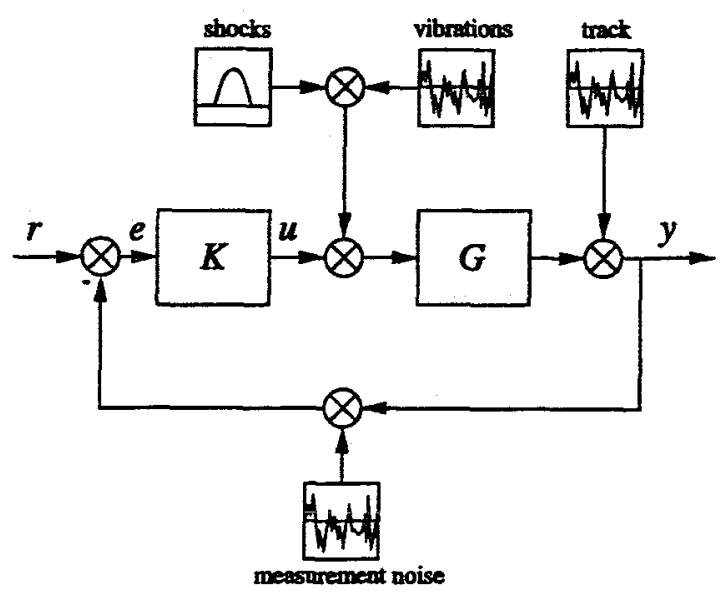

Fig. 9. Block diagram of drive servo mechanism. spot position is detected by the optical pick-up; it generates error signals (v). A $2 \times 2$ controller $K(s)$ feeds the system with the currents $u$. The (matrix) transfer function from control currents to (absolute) radial and focus spot position is indicated by $G(s)$. Mechanical shocks and vibrations are present in the input channel. The reference signal $r$ is used for seeking functionality. Measurement noise is shown in the feedback path.

The controller $K(s)$ is formed by two separate PID controllers $[6,14]$, each with the shape as in Fig. 5, thus creating two SISO control loops. This is possible because the dynamic interaction between both loops is relatively low. For the (single-speed) $C D$ drive used here, the radial loop has a bandwidth of $500 \mathrm{~Hz}$, while the bandwidth for the focus loop is $900 \mathrm{~Hz}$. These values hold for rotating singlestage mechanisms; two-stage systems both need and allow higher bandwidth. For $C D$ systems the scanning velocity of the laser spot on the disk is constant and equal to $1.3 \mathrm{~m} / \mathrm{s}$. This means that the disk itself has a variable speed, as a function of the radial position of the spot. For single-speed ( $C D$ audio) the disk rotates between $8.3 \mathrm{~Hz}$ (inner track radius $=25 \mathrm{~mm}$ ) and $3.6 \mathrm{~Hz}$ (outer track $=58 \mathrm{~mm}$ ).

The control design problem can be stated as finding a controller which achieves good tracking under disturbance conditions and given the uncertainties arising from modelling errors and plant-toplant variations from manufacturing.

In this paper we will show some new results for the focus loop (i.e. element $(2,2)$ of $G(s)$ in Fig. 9). The first (SISO) $\mu$-synthesis results for the radial loop of the CD player are described in Steinbuch et al. [30] and multivariable $\mu$-synthesis is described in Steinbuch et al. [32]. Experiences with QFT on this drive can be found"elsewhere $[9,23]$.

\subsection{Modelling}

\subsubsection{Mechanism Modelling}

- Nominal modelling. A measured frequency response of the focus actuator of the CD mechanism is given in Fig. 10. It has been determined by spectrum analysis techniques, measured under closed-loop conditions, since the actuator itself is not asymptotically stable. The procedure is to measure the sensitivity frequency response $S(\omega)=$ $(1+K(\omega) G(\omega))^{-1}$, and calculate per frequency: $K(\omega) G(\omega)=S^{-1}(\omega)-1$. Then multiplying by the inverse of the (known) controller frequency response $K(\omega)$ yields the process $G(\omega)$ [1]. 

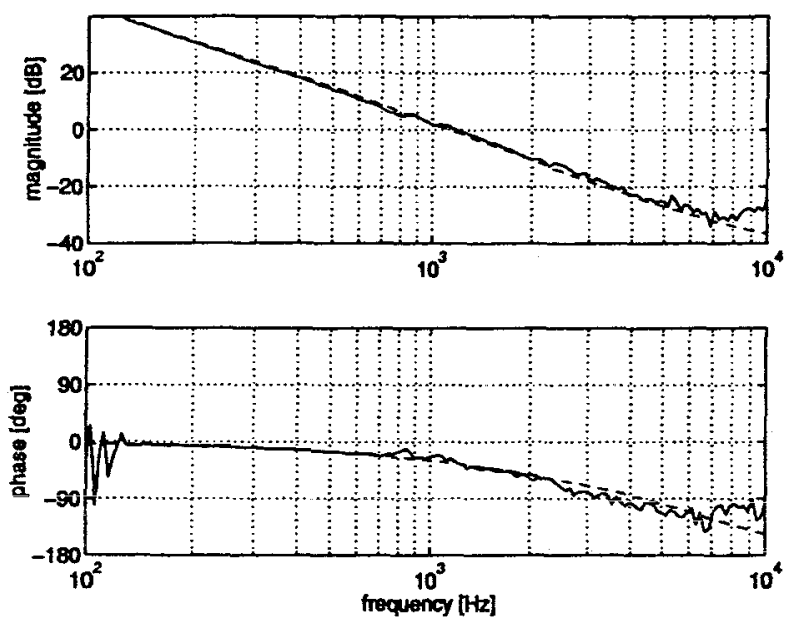

Fig. 10. Measured frequency response of the focus actuator (-), and of the identified fourth-order model (-).

The focus lens-spring system can be easily recognised as double integrator in this frequency range (with a minus sign, according to the phase). At some frequencies the measurement shows small parasitic dynamics $(800 \mathrm{~Hz})$. Notice also the phase behaviour, which is due to sampling $(26 \mathrm{kHz})$ and calculational delay $(35 \mu \mathrm{s})$. It is very important to account for this phase change, in order to design controllers with good margins (i.e. no peaking of the sensitivity).

With frequency-domain-based system identification the frequency responses have been fitted using an output error model structure with a leastsquare criterion [25] yielding a fourth-order model (see also Fig. 10). In order to improve identification results, in particular to provide the robust control design procedure with uncertainty models to capture measurement errors and modelling deviations, research is being done on robust control design-oriented parametric identification for the CD player under investigation $[7,13]$.

- Uncertainty modelling. The only system variations we want to account for in this paper are the unstructured differences between model and measurement, as additive non-parametric uncertainty $\left(\Delta_{a}\right)$. From Fig. 10 it follows that the model represents the measured frequency response reasonably well up to approximately $8 \mathrm{kHz}$. This information is used in the choice of weighting functions (see Fig. 18). For a detailed description of the parametric uncertainty modelling [18] done for the MIMO design, in order to capture manufacturing tolerances from player to player, see Steinbuch et al. [32].

\subsubsection{Disturbance Modelling}

The most important (additive signal) disturbances present in a compact disk mechanism are:

- rotation of the disk, eccentricity and track irregularities;

- mechanical vibration and shocks;

- position sensing noise.

The major disturbance source for the controlled system are eccentricity and track-position irregularities. From the $C D$ standard, all $C D$ and $C D$ ROMs may at most have a focus deflection of the disk of $1 \mathrm{~mm}$. Measurements show that at the rotational frequency of the disk this deflection is dominant, but also its higher harmonics are significantly present. In Fig. 11 a spectrum of the focus error signal measured under standard PID-controlled closed-loop conditions is shown. In order to obtain a spectrum of the disk disturbances the error spectrum should be inversely filtered by the (square) sensitivity frequency response. ${ }^{3}$ Here, we will concentrate on the error signal instead, because it will appear to be helpful during control design. The disk rotates at standard $\mathrm{CD}$ audio speed, and for a particular track this means in this case $f_{r}=4.8 \mathrm{~Hz}$ (first harmonic) and from the spectrum we can see that for this $C D$ harmonics 1,2 and 8 are strong. This varies from $C D$ to $C D$. The peak at $600 \mathrm{~Hz}$ is due to the wobble, which is a sine injected in the (radial!) loop for automatic gain control [14].

Also the frequency range around $100 \mathrm{~Hz}$ is show-

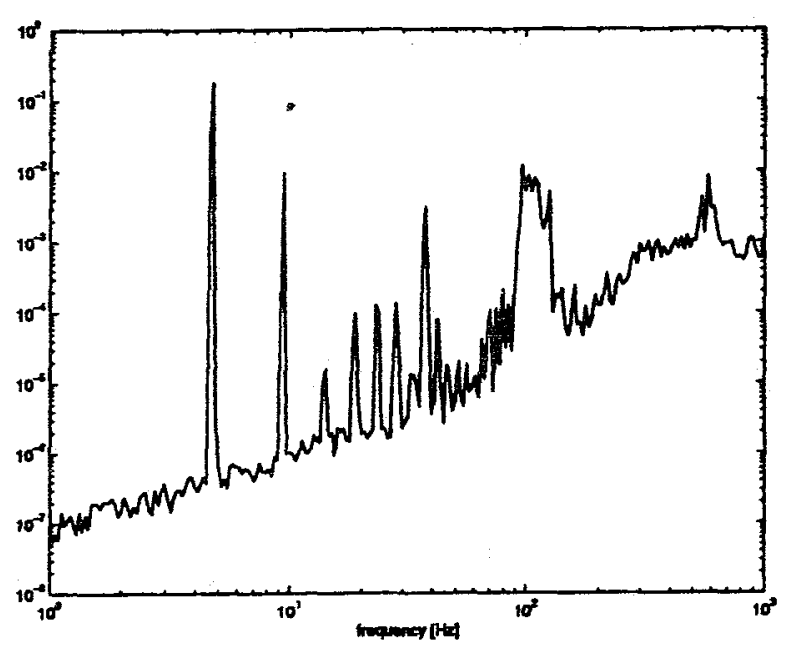

Fig. 11. Measured spectrum of focus error signal.

${ }^{3}$ Assuming that all disturbances are due to disk focus deflections, which is of course not completely true. 
ing more energy. It is a known fact that precisely in this area CDs have there first ('umbrella') flexible mode; see Fig. 12 for a finite element result (see Wortelboer [38] for numerical data of this 120st order FEM model). See McAllister [21] for similar results for HDD disks.

Besides track irregularities (disk deflections), another important disturbance source is external mechanical shocks and vibrations. Measurements show that during portable use disturbance signals occur that have a frequency content concentrated between 5 and $150 \mathrm{~Hz}$.

Finally, a completely different source of disturbance is due to measurement errors. Although one of the typical features of optical storage systems is that small particles of dust or small surface scratches on a disk are out-of-focus for the laser spot, hence do not influence tracking, large scratches of course can cause bad playability of disks. This type of disturbance can be viewed as measurement noise with a content in the higher frequency range.

\subsubsection{Performance Requirements Modelling}

The error signals in a compact disk system are generated based on optical interference principles (one-spot systems), or by differential measurements (three-spot systems). Reliable signals, within the linear range of the sensor, are guaranteed if the error remains smaller than $1 \mu \mathrm{m}$.

In addition to this requirement, seeking operations do affect the design of the actuators, but also require from the servo fast and stable (i.e. sufficiently damped) track-to-track jumps or seeks over the disk. Finally, two general requirements are (i) low power consumption of the whole system, including the mechanism and the ICs, which is very critical in portable use, and (ii) limited generation of audible noise by the actuators.

\subsection{Control Design}

\subsubsection{Motivation for Robust Control}

A good starting point for investigation into possible improvements of the servo system is to use the

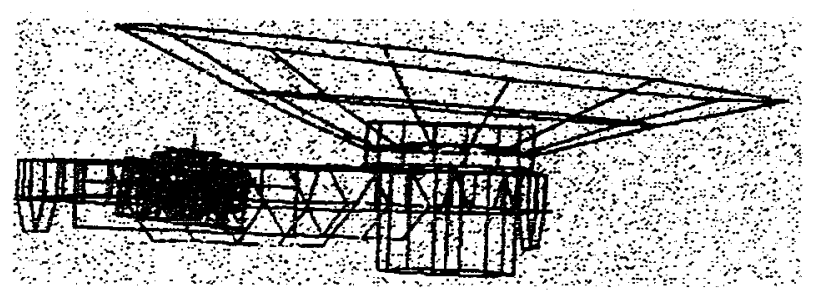

Fig. 12. First bending ('umbrella') mode of the disk $(110 \mathrm{~Hz})$. internal (PID) controller as a benchmark controller. The next step worthwhile to investigate is to perform SISO loopshaping, i.e. making PID controllers with notches etc. This is very close to using QFT [17], with the addition of quantifying robust performance. Optimal control design has the ability to automatically provide controllers which solve an optimisation problem, stated as minimising the norm of a standard plant as depicted in Fig. 1. The solution of course depends largely on which weighting filters are used as disturbance and specification modelling. A drawback is the complexity (order) of the resulting controller, being the sum of the complexity of the models of plant, uncertainties, disturbances and specification. We have done (direct) designs with fixed-order $\left(\mathrm{H}_{2}\right)$ optimal control [20], but computational complexity is high. In this paper we will show the results of indirect fixed order control design, i.e. using full order designs and afterwards applying controller order reduction.

The choice for robust control [11] is more or less straightforward if the aforementioned CD control problem is summarised as finding a controller which achieves the performance requirements under the described disturbance conditions and given the uncertainties arising from modelling errors. However, it is much less trivial how to transform time domain requirements and disturbance models (shocks, for instance) to the frequency domain for inclusion in the standard plant.

\subsubsection{Synthesis of a Standard Plant}

Having stated or modelled the plant, disturbances and specifications in Section 3.2, in this section these models will be merged into the format of a 'standard' standard plant, which set-up is applicable for a wide range of motion control problems.

The most important disturbance occurs with the disk rotational speed $\left(f_{r}\right)$, being the deflection of the disk of $1 \mathrm{~mm}$ maximal. Because the focus error should be kept smaller than $1 \mu \mathrm{m}$, the sensitivity at frequency $f_{r}$ should be less than $10^{-3}(-60 \mathrm{~dB})$. Further, pragmatically we state that for frequencies up to $150 \mathrm{~Hz}$ the sensitivity should be sufficiently small to suppress the higher harmonics of the disk eccentricity and to suppress the impact of mechanical shocks. To satisfy the requirements for high rotation speeds of the disk the bandwidth has to be increased. However, a higher bandwidth has implications for robustness of the design against manufacturing tolerances. Also, a higher bandwidth means a higher power consumption, generation of audible 


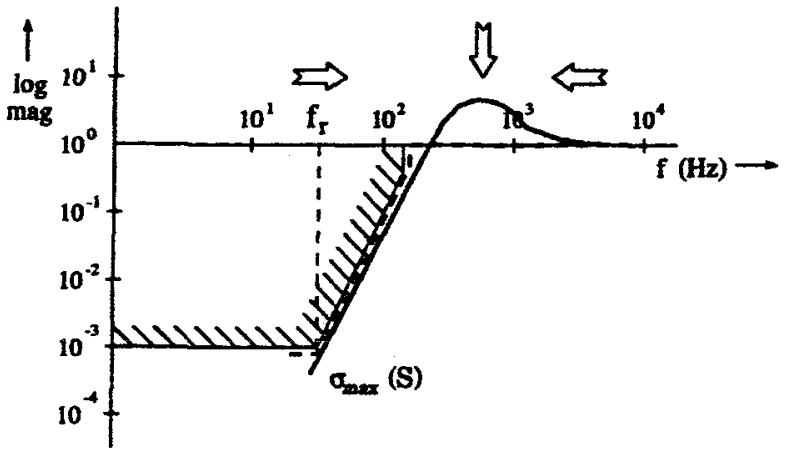

Fig. 13. Desired bound on the sensitivity function.

noise by the actuators and bad playability of disks, and more generally, increased amplification of higher-frequency disturbances. Therefore, under the required disturbance rejection we are striving towards the lowest possible bandwidth.

In the ideal situation the sensitivity function should have a form as sketched by the (bold) dashed line in Fig. 13. Because of Bode's sensitivity integral expression, the $H_{\infty}$ control design problem boils down to finding a good compromise, by using appropriate weighting functions reflecting the disturbance and specification models.

The performance specifications on the sensitivity function $S=(I+G K)^{-1}$ can be combined with the robustness specifications associated with the transfer function $K S$ accounting for the complex valued additive uncertainty $\Delta_{a}$. The weighted closed-loop block diagram used here is shown in Fig. 14. Although a more general set-up would imply also input weightings for $w_{1}$ and $w_{2}$, it is our experience that for SISO control problems good controllers can be generated using only output weightings.

The associated closed-loop (matrix) transfer function is

$$
\left[\begin{array}{l}
z_{1} \\
z_{2}
\end{array}\right]=\left[\begin{array}{cc}
W_{s} S & -W_{s} S G \\
W_{t} K S & -W_{t} K S G
\end{array}\right]\left[\begin{array}{l}
w_{1} \\
w_{2}
\end{array}\right]
$$

Weighting $W_{s}$ is used to reflect disturbance and (LF)

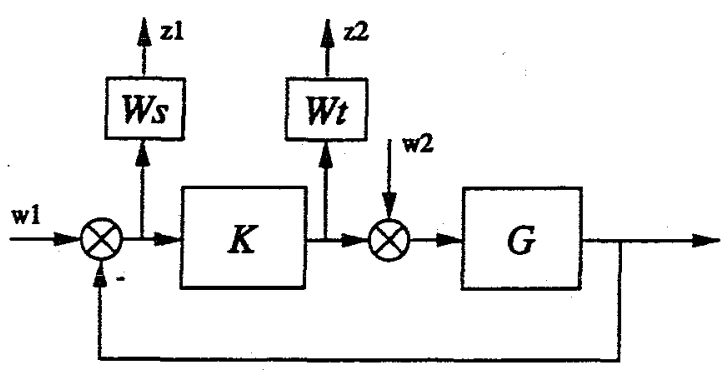

Fig. 14. Block diagram of weighted closed-loop system. performance requirements, and $W$, originates from the additive uncertainty $\Delta_{a}$ and also addresses (HF) performance requirements. Notice the equivalence with the so-called $H_{x}$-loopshaping method [16], but with larger flexibility in choosing appropriate weightings and the possibility for inclusion of parametric uncertainties. In contrast to the commonly used $(S, K S)$ design problem, it is instrumental that we have used here the additional input $w_{2}$. The main reason is that we can reduce the complexity of the problem: assume we would like to have integral action in the controller, implying an LF slope of +3 in the sensitivity. We can only enforce this by used a third-order filter for $W_{s}$ in the case of a $(S, K S)$ design. If we apply $W_{s}$ to $S G$ instead, we only need a first-order filter. This corresponds to the observation that in motion control systems we only need an integrator to achieve zero steady state error, if we have a constant (force) disturbance at the location of input $w_{2}$.

Similar arguments hold for the HF decay we would like to induce in the controller, i.e. using $K S$ instead of $\mathrm{T}=K S G$. Similar results can be obtained by using common dynamics in weighting and plant, but our experience is that this gives numerical problems. So, the standard plant of Fig. 14 and the corresponding closed-loop transfer function (1) should enable the $H_{-\infty}$-norm (i.e. gain) of $W_{\mathrm{s}} S G$ to exceed that of $W_{\mathrm{s}} S$, otherwise $S$ will approximate $W_{\text {s }}^{-1}$ instead of $S G=W_{\mathrm{s}}^{-1}$. This is achieved if the plant $G$ is scaled such that its gain equals unity at the intended bandwidth. In our experience, this scaling of the plant is extremely important to quickly generate good control designs.

\subsubsection{Disturbance 3 Based Control Design Cycle}

Based on the disturbance and performance specifications, the weighting functions are tuned. The exact location of the poles and zeros in the weights is determined in an iterative manner, such that a proper trade-off is obtained between the conflicting performance and robustness specifications. The choice of weightings appeared to be the most difficult aspect of design, since we are trying to push the performance to the achievable limits, dictated by the sensitivity integral. The performance indicator used is the cumulative power spectrum of the focus error. For a discrete time series $x(n)$, with $N$ samples, the Fourier transform is denoted by $X(\omega)$. Then the power spectral density (PSD) is defined as

$$
P_{x x}=\frac{X X^{*}}{N}
$$



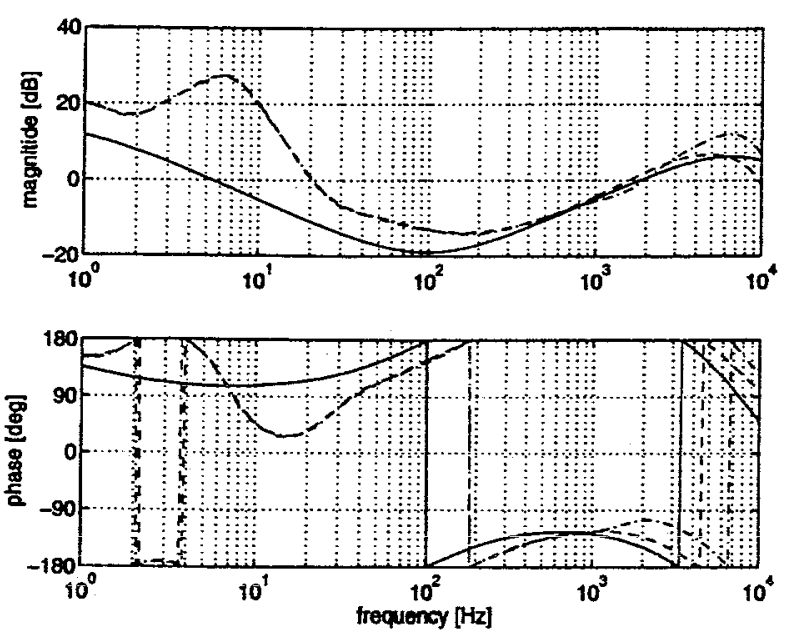

Fig. 15. Frequency responses of the PID $(-), H_{x, 1}(-)$ and $H_{\times, 2}$ $(-\cdot)$

with $*$ the complex conjugate. The cumulative power spectrum $C P_{x x}\left(\omega_{k}\right)$ is defined as

$$
C P_{x x}\left(\omega_{k}\right)=\sum_{\omega=\omega_{1}}^{\omega_{k}} P_{x x}(\omega)
$$

and

$$
C P_{x x}\left(\omega_{N}\right)=\sigma(x(n))^{2}
$$

being the variance of the time series $x(n)$.

The fine-tuning of the weights was done in a disturbance-based control design cycle with experiments within the iterative cycle:

- using the standard PID controller, the power spectral density of the focus error is measured (Fig. 11) and evaluated as a cumulative power spectrum: the steeper the slope, the more energy content at that frequency;

- an $H_{\infty}$-controller is calculated [3] using robustness $\left(W_{t}\right)$ and performance weightings $\left(W_{s}\right)$ at specified frequencies to not amplify, respectively suppress certain disturbances;

- the $H_{\infty}$ controller is implemented on the setup; and

- the error spectrum is measured and evaluated;

- when the performance does not satisfy, the weightings are adjusted, or extra weightings are added, and again a controller is calculated;

- the implementation, measurement and controller calculation steps are repeated until no further improvements are achieved.

\subsection{Implementation Results}

The DSP used is a TMS320C40 32-bit processor with floating point arithmetic. The controllers are implemented with a sampling rate of $26 \mathrm{kHz}$. Notice that irrespective of the complexity (up to a certain limit) of the controller the sampling frequency is fixed, and the delay due to digital control is equal for all controllers.

We can evaluate the achieved performance in terms of the measured frequency response of the sensitivity function, and we measure the cumulative power spectrum.

Results are presented for three (radial) track positions (BOD, MOD and EOD denoting begin, middle and end of disk, which is equal to inner track, halfway and outer track respectively) on two (out of six we used) different disks, in order to show performance robustness for variations of the disturbances. From the design cycle mentioned before, three controllers will be evaluated: a benchmark PID and two successive $H_{\infty}$ controllers.

\subsubsection{PID Controller}

Using the tuning rules from Section 2, a benchmark PID controller has been designed, with integral action and a first-order low-pass; see Fig. 15 (solid line). The bandwidth (at $0 \mathrm{~dB}$ of the loop $G K$, not plotted here) is $900 \mathrm{~Hz}$. The measured sensitivity frequency response $S(\omega)$ and complementary sensitivity frequency response $T(\omega)=K S G(\omega)$, as realised after ditigal implementation, are shown in Fig. 16 (solid lines).
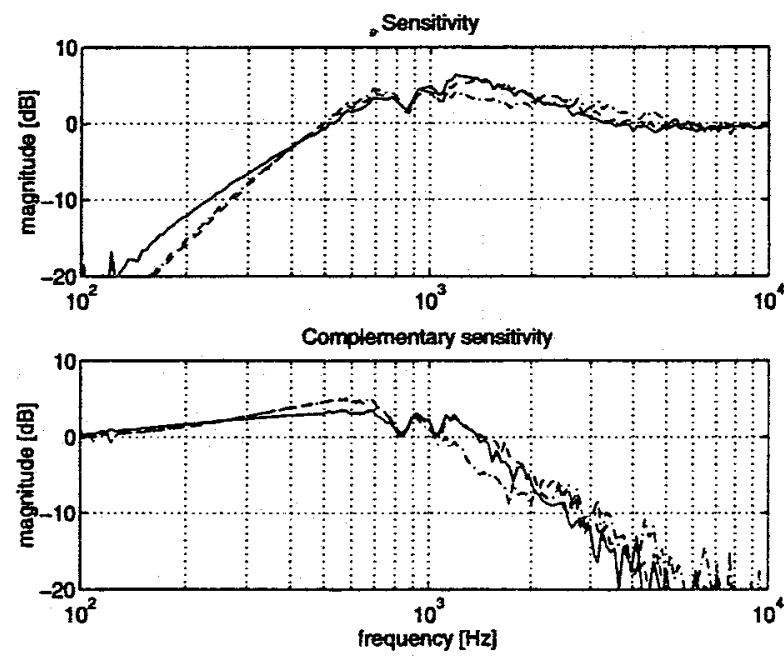

Fig. 16. Measured closed-loop frequency responses, with the PID $(-)$ and $H_{\%, 1}(-)$ and with $H_{x_{i}}(-)$. 
According to the disturbance-based control design cycle the disturbance rejection performance is evaluated using the cumulative power spectrum (see Fig. 17).

The cumulative power spectrum for the PIDcontrolled case (solid line) shows energy content in particular at the disk rotational frequency (lowest for end of disk. bottom figure). This is the case for both disk 1 and disk 2. From this experimental observation. the first $H_{x}$ controller is designed.

\subsection{2. $\boldsymbol{H}_{\boldsymbol{x}}$ Controller $\boldsymbol{H}_{x, \mathbf{1}}$}

In order to have more disturbance rejection capability at the rotational frequency of the disk, one could use very narrow (sharp) filters tuned for the rotational frequency. We will see the use of such filters in the HDD case in the next section. For CD the problem is that the rotational speed changes from 3.6 to $8.3 \mathrm{~Hz}$ (single speed), and is not (always) available as signal. In Dötch and Smakman [12] and Steinbuch and Schootstra [29] repetitive control has been applied, accounting for changes in the periodicity. In this paper we will adopt a robust solution by having a broad weighting filter to obtain reduction.

The performance weighting $W_{s}$ has a basic firstorder low pass to ensure integral action, and in addition $(2 \times$ second-order $)$ dynamics to obtain extra rejection at low frequencies (see Fig. 18, upper plot). For this $H_{x, 1}$ design, the weighting $W_{t}$ is designed as a second-order high-pass filter, in order
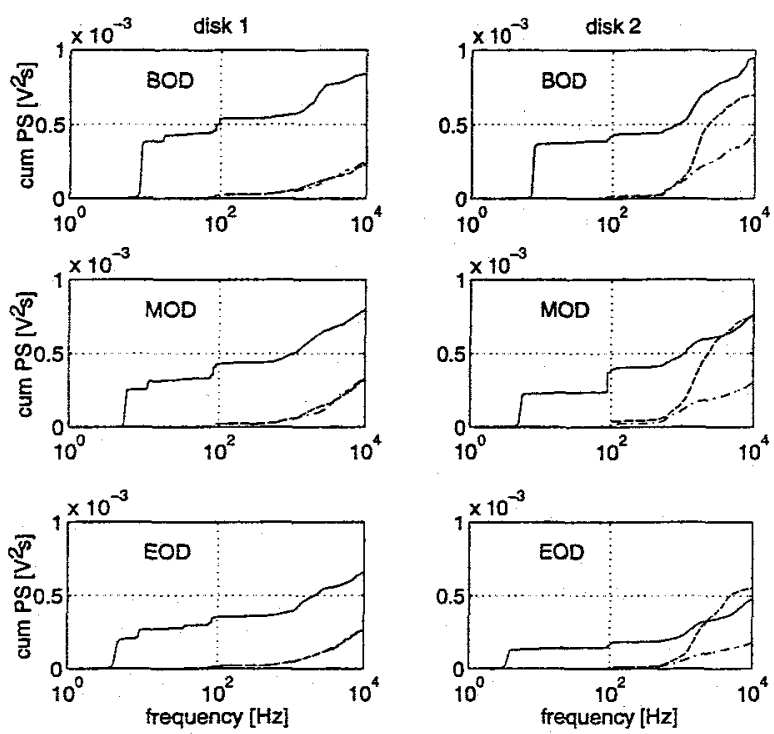

Fig. 17. Cumulative power spectrum with PID (-), $H_{x, 1}(-), H_{x, 2}$ (-), for disk I (left), disk 2 (right), for the begin, middle, and end position on the disks.
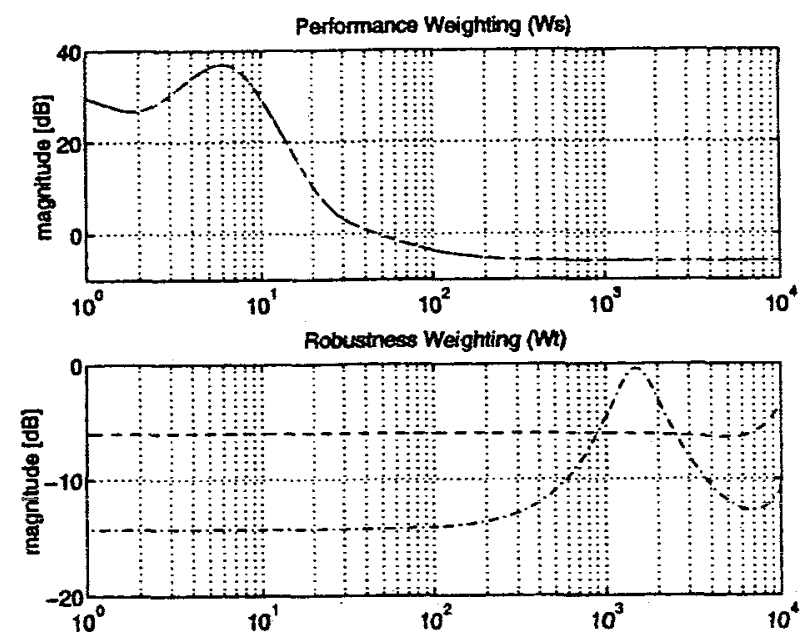

Fig. 18. performance $W_{s}$ (upper) and Robustness $W_{t}$ (lower) weightings for $H_{x}$ controller $H_{x, 1}(--)$, and $H_{x, 2}(-)$.

to enforce a -2 decay in the controller $K$. Its breakpoint is at $8 \mathrm{kHz}$ (Fig. 18, lower plot, dashed line), to achieve robustness for unmodelled dynamics and model errors (recall Fig. 10), but aiso to reflect requirements for low controller gains at high frequencies. The calculated controller $H_{x, 1}$ has order 10 (plant $4+W_{s} 5+W_{t} 2=11$, then reduced). In Fig. 15 the frequency response $H_{x, 1}(\omega)$ is shown as a dashed line. Indeed, up to almost $600 \mathrm{~Hz}$ the gain is higher than the gain of the PID controller, and the $H_{s, 1}$ controller has significant gain at low frequencies, while the gain at the intended bandwidth of $900 \mathrm{~Hz}$ has not changed, indicating that we achieved this with equal bandwidth. Notice that of course a trained 'loopshaper' could have tuned a similar controller as well. However, the benefit of using optimal control here is that the controller synthesis is done automatically and is reproducible. In Fig. 16 the measured sensitivity and complementary sensitivity are shown for the $H_{x, 1}$ controlled case as well. Notice the improved reduction capability up to $400 \mathrm{~Hz}$ (upper plot).

Again the performance assessment is done using the cumulative power spectrum (see Fig. 17 with the dashed line). Obviously, the additional LF disturbance rejection capability has a major impact on the focus error, especially for disk 1 . Evaluating the results for disk 2, it is visible in Fig. 17 (right column) that above $1 \mathrm{kHz}$ a lot of the profit gained by the $H_{x, 1}$ controller is lost. For this reason we iterate a second cycle. 


\subsection{3. $\boldsymbol{H}_{\infty}$ Controller $\boldsymbol{H}_{\infty, 2}$}

In order to improve the behaviour above the bandwidth, a (second-order) filter is added to the robustness weighting $W_{t}$, not because of model uncertainties but because of the disturbance properties. This additional filter has high gains around the problematic area (from 1 to $2 \mathrm{kHz}$ ); see Fig. 18 (dashed/dotted line). Due to a normalising scaling applied to the weightings, at other frequencies the gain has changed (but the additive uncertainties above $8 \mathrm{kHz}$ are still captured). The calculated controiler $H_{x .2}$ has again (!) order 10 (plant $4+W_{s} 5$ $+W_{t} 4=13$, then reduced using closed-loop model reduction [38]). In Fig. 15 the frequency response $H_{\infty, 2}(\omega)$ is shown as dashed/dotted line. There is a different behaviour around $2 \mathrm{kHz}$, but the gain at $900 \mathrm{~Hz}$ has not changed, indicating that we again achieved this with equal bandwidth. In Fig. 16 the measured sensitivity and complementary sensitivity are shown for the $H_{x, 2}$-controlled case as dashed/dotted lines. In these plots the benefit of the additional $W_{t}$ weighting is clearly visible as lower $\mathrm{S}$ and $\mathrm{T}$ gains in the range $1-2 \mathrm{kHz}$. The cumulative power spectrum for this controller is shown in Fig. 17 (dashed/dotted). Disk 2 shows a significant improvement, while disk 1 is still good. Based on this cumulative power spectrum we decided to stop the design cycle, since little further improvement can be gained without increasing bandwidth (taking into account the cost of power, robustness etc.).

The final determination of the disturbance rejection performance is made in time-domain, ${ }^{4}$ using the probability density function of the focus error (see Fig. 19) for all three controllers. The $3 \sigma$ values of the time records of the focus error signals are as follows: if the PID-controlled situation is normalized as $100 \%$, then the $H_{\infty, 1}$ gives values for disk $13 \sigma$ $=52,65$ and $65 \%$, for BOD, MOD and EOD position on the disk respectively. For disk 2 these values are $3 \sigma=86,99$ and $107 \%$. For $H_{x, 2}$ these values are: for disk $13 \sigma=54,65$ and $64 \%$, and for disk $23 \sigma=70,67$ and $68 \%$. With the second design the profit is about $30 \%$.

The results show the improvement we can obtain by adding dynamics at specific disturbance-related places in the controller, while keeping the same bandwidth.

\footnotetext{
${ }^{4}$ That is, where we usually live in, both human beings and
} control systems.
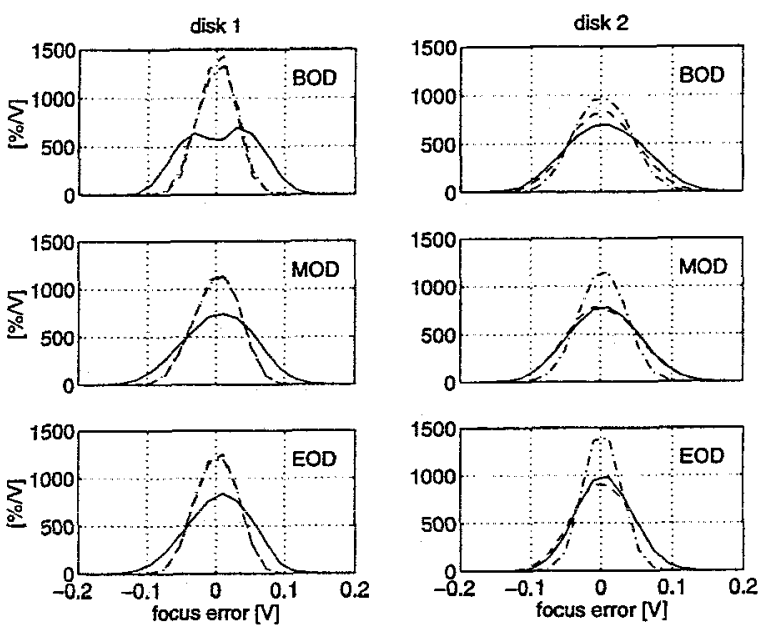

Fig. 19. Probability density function of the focus error, PID (-), $H_{x, 1}(-)$ and $H_{x .2}(-)$ controller, for disk 1 (left), disk 2 (right), for the begin, middle and end position on the disks.

\section{Magnetic Disk Drive Control}

\subsection{Servo Control Problem}

The control of hard disk drives attracted reasonable attention in the literature $[8,10,33]$. The characteristics are similar to the $C D$ servo problem, with main differences that the disk is non-removable, implying also much lower eccentricity, and has a fixed and higher rotation rate. Furthermore, the actuator is single degree of freedon, i.e. only radial tracking. Finally, most high-performance drives today are sectored (embedded) servo drives, resulting in a relatively low sampling rate of the servo information, hence limiting performance.

Consider the schematic representation of a hard disk drive in Fig. 20. The main elements that can

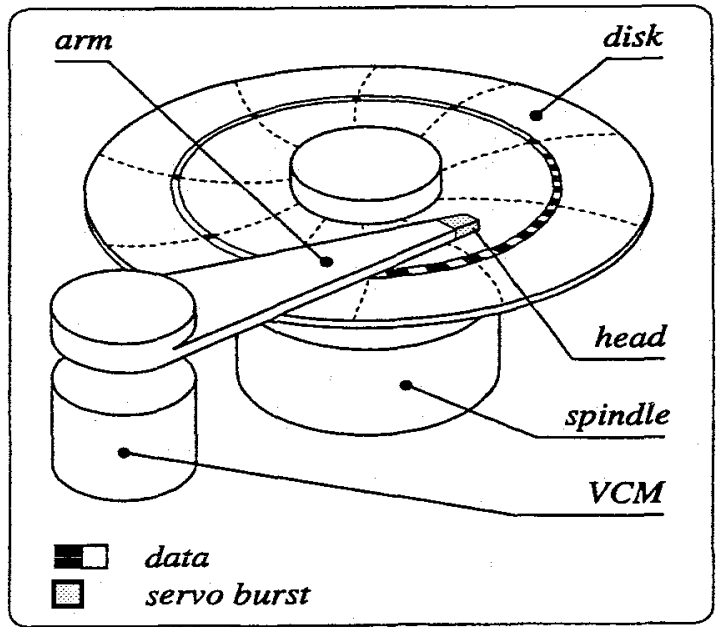

Fig. 20. Schematic representation of hard disk drive. 
be distinguished are the magnetic read/write head, the rotating (non-removable) disk, the arm and the VCM (voice coil motor). Fixed servo bursts written on the disk provide information on the deviation of the head from the centre of a track. The sampling frequency of the resulting discrete time system is physically determined by the rotational speed of the disks and the number of servo bursts written on each track. The drive under investigation has a rotational speed of $120 \mathrm{~Hz}(7200 \mathrm{rpm})$ and a sampling frequency of $8 \mathrm{kHz}$. The block diagram depicted in Fig. 9 shows the tracking control loop, as in the compact disk case (but for the HDD in SISO form).

The hard disk drive under investigation is a highend device, with a capacity of $9 \mathrm{~GB}$ in a 3.5 -inch housing. It has a package of 10 disks (see Fig. 21).

In the next sections the same procedure as shown for the Compact Disk mechanism, will be shown for the tracking problem of the HDD.

\subsection{Modelling}

\subsubsection{Hard Disk Drive Modelling}

In Fig. 22 a measurement of the (VCM) actuator is shown. As with the $C D$ problem, we are faced with a basic -2 slope for the rigid body dynamics, and some higher harmonics from parasitic dynamic vibrations. Notice also the significant impact of sampling and calculational delay on the phase of the measurement. The spike at $120 \mathrm{~Hz}$ is a bad measurement due to the working of an adaptive sinecanceller in the drive's DSP for suppressing the eccentricity. The measurement was used to fit a 10 -

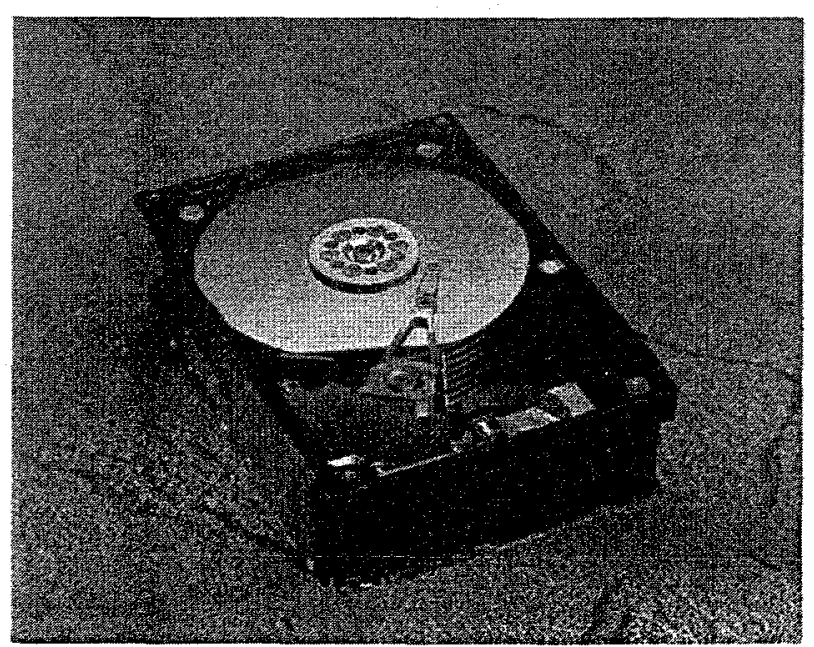

Fig. 21. Hard disk drive under investigation.
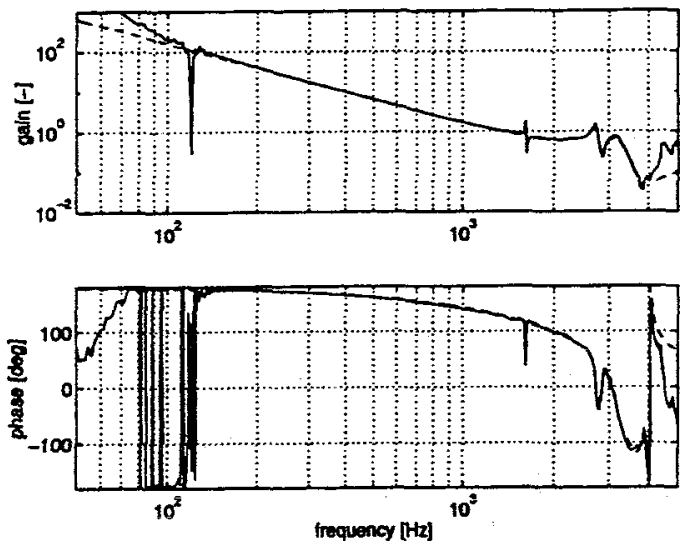

Fig. 22. Tenth-order fit (--) on measurement $(-)$ of the hard disk drive actuator.

order LTI model, whose frequency response also is plotted in Fig. 22.

\subsubsection{Disturbance Modelling}

In hard disk drives some distinguishable disturbance sources $[2,22,24]$ are 'motion' of the disks, due to ball bearings and eccentricity of the disks, position sensing noise, ADC and DAC noise, power amplifier noise, windage (caused by air flow generated by the spinning disks), and finally mechanical vibration and shocks. In this paper we will concentrate on the motion of the disks.

\subsubsection{Performance Requirements Modelling}

The amplitude of the error (called position error signal, or PES) determines the achievable amount of tracks per inch: the smaller the error, the closer the tracks can be put together. This is a strong technological trend exploited by all hard disk drive manufacturers, where it is possible to evolve more rapidly than within the optical storage area, because individual companies can push their own limits, since compatibility is not an issue due to the fixed disks. Specifications on the error and the standard deviation of the error are respectively:

$$
\begin{aligned}
|e(t)| & \leq 15 \% \text { trackwidth, } \forall t \\
3 \times \sigma_{\mathrm{e}} & \leq 4 \% \text { trackwidth }
\end{aligned}
$$

where typically the $l_{x}$ bound on $e$ is the worst case, i.e. in the case of mechanical shocks, while the variance requirement holds for normal operation. 


\subsection{Control Design}

In this section the following linear controllers are compared: the internal controller; a PID/notch controller; and an $H_{\infty}$ controller.

The internal controller has a PID structure, with in addition two adaptive sine-canceling schemes $[5,34]$ to suppress the first and the eighth harmonic of the rotation of the disks (these disturbances are called RRO: repeatable runout). The control systems bandwidth (still defined as the $0 \mathrm{~dB}$ crossing of the open-loop frequency response) is around $450 \mathrm{~Hz}$. The sampling frequency of the controller is $8 \mathrm{kHz}$.

The PID/notch controller is implemented on the same DSP as used for the CD player. The sampling frequency of the controller is also $8 \mathrm{kHz}$. The $\mathrm{PID} /$ notch controller has the same poles and zeros as the internal controller. At $120 \mathrm{~Hz}$ an inverse notch is placed to suppress the relatively large eccentricity disturbance of the disks. Notice that in contrast to the $\mathrm{CD}$ case the inverse notch (sine generator) at the rotational frequency can be very sharp, because the disk speed is constant (although a sharp secondorder filter has a relatively slow transient behaviour which may give problems with seeking).

The $H_{\infty}$ controller was designed using the same disturbance-based control design cycle as in the $\mathrm{CD}$ case: starting with the PID/notch controller, the PES spectrum is measured and evaluated as a cumulative power spectrum, an $H_{\infty}$ controller is calculated using robustness $\left(W_{t}\right)$ and performance weightings $\left(W_{s}\right)$ at specified frequencies to not amplify, respectively suppress certain disturbances, the $H_{\infty}$ controller is implemented and the spectrum of PES is measured and evaluated again, the weightings are adjusted, or extra weightings are added, and again a controller is derived, implemented etc. These steps are repeated until no further improvements are achieved.

In Fig. 23 the weightings used during the $H_{\infty}$ controller design are plotted, and in Fig. 24 the frequency response functions of the $H_{\infty}$ controller and the PID/notch controller are plotted.

Although all controllers will be compared with each other, the internal controller has a certain advantage because it has less computational delay (due to faster DAC and $A D C$ and the use of oversampling): this causes some phase advance at higher frequencies. It would be more justifiable just to compare the two external controllers.

\subsection{Implementation Results}

The controllers are rated on their ability to suppress noise and shocks. In this paper we will show the
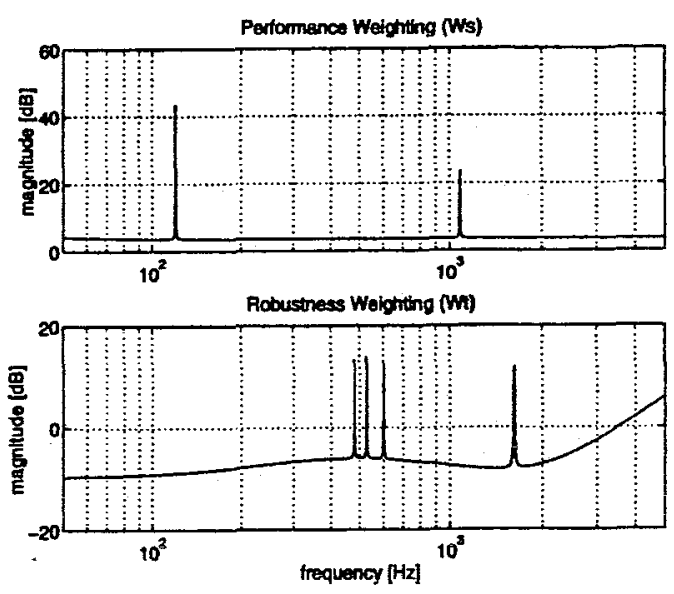

Fig. 23. Performance (upper) and robustness (lower) weightings for $H_{x}$ controller design.
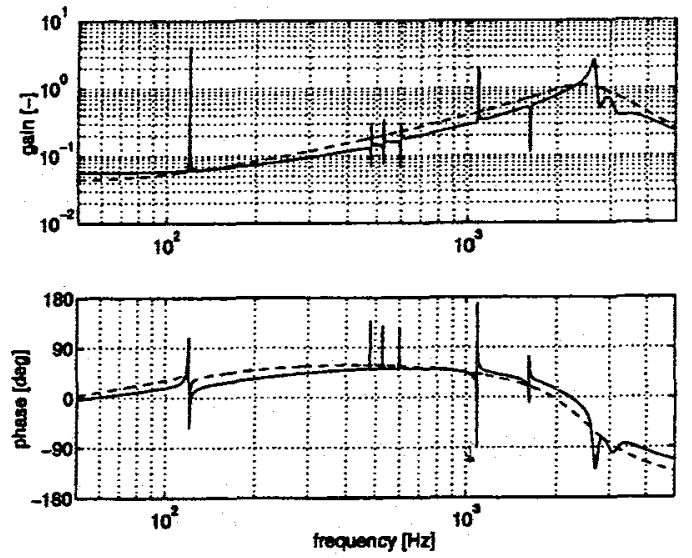

Fig. 24. Frequency response functions of the $H_{x}$ controller (-) and the PID/notch controller (--).

results for noise. The $3 \sigma$ value should be smaller than $4 \%$ of a track. In Fig. 25 the PES with the three different controllers is plotted.

The $3 \sigma$ values that classify the three controllers are: $3 \sigma_{\text {internal }}=4.22 \%, 3 \sigma_{\mathrm{PID} / \text { notch }}=4.45 \%, 3 \sigma_{H x}$ $=3.56 \%$. Compared to the internal controller the implemented $H_{\infty}$ controller performs about $15 \%$ better, and compared to the PID/notch controller even $20 \%$ better. The cumulative power spectrum is plotted in Fig. 26. From this figure, we see that the internal controller performs better in the lower frequency range, up to $500 \mathrm{~Hz}$. From 500 to $1000 \mathrm{~Hz}$, the internal and the PID/notch controller perform worse than the $H_{\infty}$ controller. At $1080 \mathrm{~Hz}$ the $H_{\infty}$ controller is also better than the other controllers. As a summarising conclusion we can state that the disturbance-based control concept, with implementation inside the design cycle of tuning weights, 

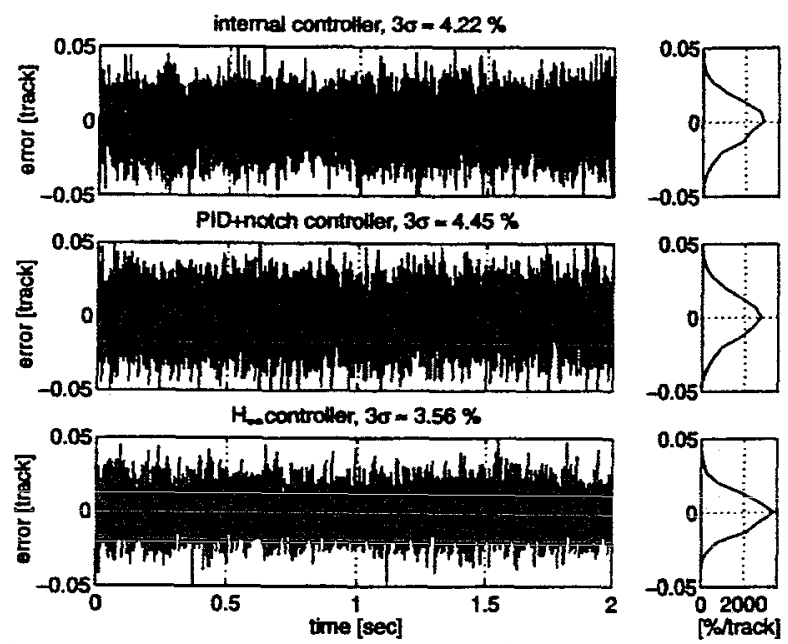

Fig. 25. Position error signal (PES) of internal, PID/notch and the $H_{x}$ controller.

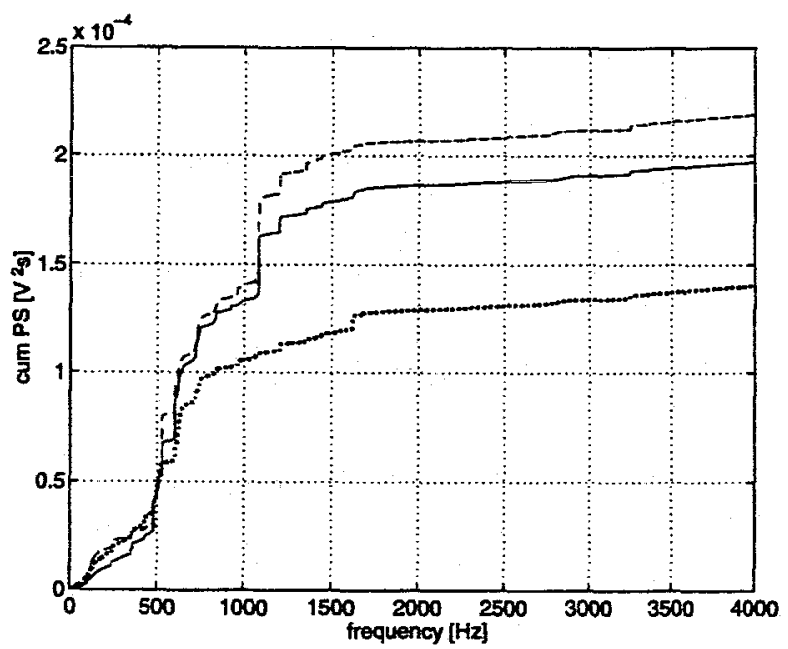

Fig. 26. Cumulative power spectrum of internal (-), PID/notch $(--)$ and the $H_{\infty}$ controller (...).

has improved the tracking performance of the hard disk drive.

\section{Challenges for Research}

In the previous sections we have shown how we have applied robust control to information storage drives. Based on our experience, in this section we will first answer the question of relevance of robust control methodology for industry (Section 5.1) and from this viewpoint we will extract possibilities for future research directions (Section 5.2).

\subsection{Industrial Relevance ...}

From our industrial perspective, robust control methods such as $\mathrm{H}_{x}$ and $\mu$-synthesis ${ }^{5}$ provide a framework to state and solve control problems, in particular providing a natural extension from classical control, closed-loop orientation, design formalism, and inclusion of parametric uncertainties.

- Natural extension from classical control. The standard procedure within industry of designing controllers for motion servo systems, such as the CD and HDD in this paper, is to use loopshaping, as was explained in Section 2. The frequency domain-based design rules have been first extended to include parametric uncertainties and closed-loop specifications by QFT [17]. The development of $H_{\infty}$ and $\mu$-synthesis has given a theoretically thorough foundation as a formal optimisation theory for the design of robust controllers. As a parallel result, but not less important, the notion of standard plant emerged also for the $\mathrm{H}_{2}$ case, from which the LQG methodology can now be stated in terms of a classical frequency domain viewpoint.

- Closed-loop orientation. In contrast to the openloop oriented loopshaping of controllers, a feature of (modern) robust control is that specifications are stated in terms of closed-loop requirements. In addition, the (equal) role of disturbances and specifications is formalised. This is important for industry, since it provides a new paradigm for control system design. As an example, take the (common!) motor-controlled case of Section 2 but where specifications are on the (non-measurable) load-positioning accuracy. This cannot be systematically dealt with using classical SISO loopshaping techniques.

- Design formalism. The control engineers' ${ }^{3}$ capability to do a good task in loopshaping remains an important requirement for successful control engineering in practice. ${ }^{7}$ However, automated reproducible procedures will become more and more important with the growing complexity of systems and their (embedded) software.

- Inclusion of parametric uncertainties. Last but not least the notion of robustness for parametric uncertainties is certainly not new, and control

\footnotetext{
5 And also QFT but with a different flavour.

${ }^{6}$ It is more than only instructive if academic researchers also can do a good job in loopshaping, before they start using advanced, automated control design techniques!

${ }^{7}$ We have seen that, although the design procedure is ready, still experience is needed to do good control design.
} 
engineers in industry are fully aware of the nonrobustness of making sharp notches within their filters. However, there is a need for synthesis techniques in which specified uncertainty bounds are taken care of automatically, as in $\mu$-synthesis.

Given these benefits, does industry have a positive profit/cost balance for robust control? Not yet, to our belief, for two reasons. First, profit is too low, because most systems are being designed as a SISO system simply because too few people designing new systems are aware of potential benefits of MIMO control, ${ }^{8}$ and for SISO systems the profit suffers from limited awareness and tools for disturbance modelling. Secondly, costs are too high, because setting up a good standard plant problem takes more time than doing a good SISO loopshape design.

In order to improve the profit/cost balance, and based on our experience with modern control methods, parts of which are described in this paper, we would like to emphasise areas for future research in robust control as seen from an industrial perspective.

\section{2. ... of Good Setpoints for Academic Research}

In our opinion the important issue of design of control systems is largely underemphasised in academic research. By design we mean all aspects of modelling the plant, uncertainties, specifications, disturbances, making choices for signal sets, norms, controller structures, operating point, reduction, implementation, discretisation, i.e. the whole process of arriving at a formal control problem. This is in contrast to the majority of publications within our field, covering a wide range of solutions to (nice and solvable) mathematical problems.

From an industrial viewpoint it would be more than welcome to have access to a handbook of design examples, and/or have available a strategy for robust control design. In order to stimulate academia to pursue this thought, it is our belief that it is not enough to have nice 'lab' experimental setups for students to work on, but merely it is of importance to pose real challenging target specifications!

Specific items deserving more attention in academic research are described below, concerning

\footnotetext{
${ }^{8}$ Notice that this motivates research in the area of simultaneous design of the system and its control functions; see for instance Shi and Skelton [27].
}

modelling, performance, implementation, tools, transferability and theory.

- Modelling. The confrontation with real applications showed us the lack in literature to cope with uncertainty modelling. Some extensions relevant for application have been developed [18] but remaining open questions are: (i) how to detect which uncertainties are relevant and which are not, i.e. the question of uncertainty reducibility [4], (ii) how to extract from an experimental setup the knowledge about the true variations of system parameters, and (iii) nominal and uncertainty modelling for mass products, i.e. multimodel identification. In addition, more tools and experiences should be published about frequency domain identification, i.e. how to generate good models, based on frequency response measurements.

Important research directions are currently driven by the progress made in identification for robust control $[15,35]$. The role of disturbance identification is currently handled within the standard frameworks of $H_{2}$ and $H_{s}$-related signals, either as noise or as worst-case deterministic disturbances. However, to really 'squeeze Bode's sensitivity' less general descriptions of disturbances are needed. Moreover, there is a need to have methods to obtain from experimental data good, non-conservative models or model sets for disturbances.

- Implementation. The application area of highvolume electronics (CD, HDD, tape) strongly constrains the complexity of the controller, being the main motivation for the research we have put into model reduction techniques [38]. In contrast to the existing literature we have experienced the importance not only to look for error bounds or optimality, but to make use of the control engineers' knowledge on the closed-loop relevance of controller dynamics within the model reduction framework.

- Performance. Although the frequency domain provides strong analysis results, the real specifications and disturbances are in the time domain. In particular, the combination of (co)variance requirements, worst-case signals and hard bounds on signal amplitudes is what is needed to really push the 'limits of performance'. For this reason, the current interest in $l_{1}$ robust control, or more general multi-objective control [26], is of great importance.

- Tools. Most applications presented in the literature are modelled with relatively low-order models 
and simple uncertainty descriptions. For such problems all tools work well. However, the real benefit from using advanced control only appears when either truly MIMO problems or SISO problems with severe specifications, and for which a large amount of attention is paid to modelling the dynamics of the system and disturbances, are being dealt with. However, for such systems, like the $\mathrm{CD}$ and HDD application described in this paper. a lot of numerical problems occur and have to be solved [19]. The solutions are not trivial. and not well documented in the literature.

- Transferability. The theory and methodology of robust control is hard to transfer to control engineers working in industrial development groups, not only because of the previous remarks, but also because of the way theory is explained. Also, it is not straightforward how to set up a standard plant and to design (and scale!) weights. For this reason there is a need to develop a set of easyto-use software tools, with as few tunable design parameters as possible, combining (classical) loopshaping experience and robust control theory, including numerical conditioning and closed-loop model reduction.

- Theory. Last but not least, of course every real control design challenge involves multi-objective criteria, including discrete-event operational conditions (e.g. start/stop), where fixed-order controllers are required, and with changing operating conditions. Also, the field of non-linear control (even for linear systems) is still underdeveloped. Hence, there is a lot of work to do.

\section{Conclusions}

In this paper, we have shown a 'standard' industrial feedback tuning, as being used for motion control systems. This servo design is based on manual loopshaping according to some design rules, and serves as a benchmark for investigation into the application of robust control to information storage systems. New results have been obtained for the focus control loop of a compact disk player and radial tracking of a hard disk drive. The most difficult aspect of design appeared to be the shaping of weighting functions such that a proper trade-off is obtained between the conflicting performance and robustness specifications. A disturbance-based control design iteration was introduced, where we put experiments within the design cycle. Experimental results show improved performance with respect to disturbance attenuation.
In order to improve the profit/cost balance of robust control, and based on our experience with the application of robust control to (mechanical) servo systems, we have emphasized areas for future research in robust control being our industrial feedback to academia.

\section{Acknowledgements}

The authors would like to thank the former Disk Memory Division of Hewlett-Packard Company, Boise, for their help and for the possibility to work on their latest and last drives. Unfortunately, in 1996 Hewlett-Packard withdraw as a HDD manufacturer. The authors would also like to thank Danny Abramovitch, Hewlett-Packard Labs, for helpful suggestions, and Jean-Christophe Boissy, Philips Research Labs Paris and Ewout Rotte, Philips Semiconductors for their contribution in the HDD project. The Optical Recording Development group of Philips Components is gratefully acknowledged for their support and cooperation, and because they serve as our industrial benchmark. Finally, the authors would like to thank the Mechanical Engineering Systems and Control Group, Delft University of Technology, headed by Professor Bosgra, for the fruitful cooperation we have.

\section{References}

1. Abramovitch D. The Banshee multivariable workstation: a tool for disk drive servo research. ASME Adv Inform Storage Syst 1993; 5

2. Abramovitch DY, Hurst T, Henze D. An overview of the PES Pareto method for decomposing baseline noise sources in hard disk position error signals. IEEE Trans Magnetics 1998; 34: 17-23

3. Balas GJ, Doyle JC, Glover K, Packard AK, Smith R. $\mu$-Analysis and synthesis toolbox. Musyn, Minneapolis 1991

4. Beck CL, Doyle J, Glover K. Model reduction of multidimensional and uncertain systems. IEEE Trans Autom Control 1996; 41(10): 1466-1477

5. Bodson M, Sacks A, Khosla P. Harmonic generation in adaptive feedforward cancellation schemes. IEEE Trans Autom Control 1994; 39(9): 1939-1944

6. Bouwhuis G. et al. Principles of optical disk systems. Adam Hilger, Bristol 1984

7. Callafon RA de, van den Hof PMJ, Steinbuch M. Control relevant identification of a compact disk pickup mechanism. In: 1993 IEEE conference on decision and control, San Antonio, December 1993, pp 20502055

8. Chew KK. Control system challenges to high track density magnetic disk storage. IEEE Trans Magnetics 1996; 32(3): 1799-1804 
9. Chait Y, Park MS, Steinbuch M. Design and implementation of a QFT controller for a compact disk player. J Syst Eng 1994; 4: 107-117

10. Corliss JM. Neubert R. DSP helds keep disk drives on track. Computer Design 1988: 15 June: 60-65

11. Doyle JC, Glover K, Khargonekar P. Francis BA. State-space solutions to standard $H_{2}$ and $H_{x}$ control problems. IEEE Trans Autom Control 1989; 34: $831-847$

12. Dötch HGM. Smakman HT, Van den Hof PMJ, Steinbuch $M$. Adaptive repetitive control of a compact disc mechanism. In: 1995 IEEE conference on decision and control. December 1995. pp 1720-1725

13. Dötsch HGM, Van den Hof PMJ, Bosgra OH, Steinbuch $M$. Identification in view of control design of a CD Player. Selected topics on Identification, Modelling and Control. Delft 1996; 9: 25-30

14. Draijer W, Steinbuch M, Bosgra $\mathrm{OH}$. Adaptive control of the radial servo system of a compact disk player. IFAC Autom 1992: 28: 455-462

15. Gevers M. Towards a joint design of identification and control? In: Trentelman HL, Willems JC (eds). Essays on control: perspectives in the theory and its applications. Birkhäser, Boston. 1993. pp 11 1-151

16. Glover K, McFarlane D. Robust stabilization of normalized coprime factor plant descriptions with $H_{x^{-}}$bounded uncertainty. IEEE Trans Autom Control 1989; 34: 821-830

17. Horowitz I. Survey of quantitative feedback theory (QFT). Int J Control 1991; 53: 255-291

18. Lambrechts P, Terlouw JC, Bennani S, Steinbuch M. Parametric uncertainty modeling using LFTs. In: 1993 American Control Conference, 1993, pp 267-272

19. Laub AJ, Patel RV, Van Dooren PM. Numerical and computational issues in linear control and system theory. In: Levine WS (ed). The control handbook. CRC Press, Boca Raton, FL. 1996. pp 399-414

20. Levine WS, Athans M. On the determination of the optimal constant output feedback gains for linear multivariable systems. IEEE Trans Autom Control $1970 ; 15(1): 44-48$

21. McAllister JS. Characterization of disk vibrations on aluminum and alternate substrates. IEEE Trans Magnetics 1997; 33(1): 968-973

22. Ohmi T. Non-repeatable runout of ball-bearing spindle motor for 2.5" HDD. IEEE Trans Magnetics 1996; 32(3): $1715-1720$

23. Park MS, Chait Y, Steinbuch M. Inversion-free design algorithms for multivariable quantitative feedback theory: an application to robust control of a CD-ROM. IFAC Autom 1997; 33(5): 915-920
24. Radwan HR. Phan DT. Cao K. Effect of disk drive actuator unbalance on track following response to external vibration and shock. IEEE Trans Magnetics 1996: 32(3): 1749-1755

25. Sanathanan CK, Koerner J. Transfer function synthesis as a ratio of two complex polynomials. IEEE Trans Autom Control 1963; 8: 56-58

26. Scherer C. Mixed $H_{2} / H_{\infty}$ control. In: Isidori A (ed). Trends in control: a European perspective. SpringVerlag. Berlin. 1995. pp 173-216

27. Shi G. Skelton RE. An algorithm to integrate passive and active control. In: 1996 IEEE international symposium on computer-aided control system design, 1518 September 1996, Dearborn, pp 536-541

28. Stan SG. Baky JL. Adaptive speed algorithms for CDROM systems. IEEE Trans Consumer Electron 1996: $42(1): 43-51$

29. Steinbuch M, Schootstra G. Robust and adaptive repetitive control. Journal A 1996; 37: 9-15

30. Steinbuch M, Schootstra G, Bosgra OH. Robust control of a compact disk player. In: IEEE 1992 conference on decision and control, Tucson, AZ, 1992. pp 2596-2600

31. Steinbuch M, Smit SG, Schootstra G, Bosgra OH. $\mu-$ Synthesis of a flexible mechanical servo system. In: 1991 American control conference, Boston, MA, 2628 June 1991, pp 593-598

32. Steinbuch M, van Groos PJM, Schootstra G, Wortelboer PMR. Bosgra OH. $\mu$-Synthesis for a compact disk player. Int J Robust Nonlinear Control 1998; 8: 169-189

33. Teo YT, Tay TT. Application of the $1^{1}$-optimal revolution strategy to a hard disk servo system. IEEE Trans Control Syst Technol 1996; 4 (4): 467-472

34. Tomizuka M. Zero phase error tracking algorithm for digital control. Trans-ASME J Dynamic Syst Measure Control 1987; 109: 65-68

35. Van den Hof PMJ, Schrama RJP. Identification and control: closed loop issues. IFAC Autom 1995; 31(12): $1751-1770$

36. Wie B, Bernstein DS. Benchmark problems for robust control design. In: 1991 American control conference. Boston, MA, 26-28 June 1991, pp 1929-1930

37. Willems JC. On interconnections, control, and feedback. IEEE Trans Autom Control 1997; 42(3): 326339

38. Wortelboer P. Frequency weighted balanced reduction of closed-loop mechanical servo-systems: theory and tools. PhD thesis, Delft University of Technology, Delft, 1994 


\section{Discussion on: 'Advanced Motion Control: An Industrial Perspective' by M. Steinbuch and M. L. Norg}

\section{Discussion by D. Y. Abramovitch}

This paper gives an excellent quasi-survey of area of control for storage systems and how advanced control techniques can be applied. It is worth elaborating on a few of the points made in the paper from the perspective of this author's own limited experience.

The first point is that a complete design using only modern analysis and design techniques is a rare case in the storage industry. This is partly because of the difficulty (mentioned in the paper) of posing the industrial problem in modern frameworks. However, a compounding factor is that of time constraints on design time. Any design method which requires the controls engineer to use different techniques in every aspect of the drive design is bound to take far more time to implement than making marginal improvements on a current design method. ${ }^{2}$ Thus, controls engineers - sensing that their performance evaluations are on the line - are very cautious about deviating from practices that have worked before.

It has been this author's experience that the researcher stands a much better chance of having a new idea implemented into a storage system if it can be dropped as a piece into an existing design methodology. A perfect example of this is in the area of adaptive feedforward cancellation schemes $[5,11]$ which were introduced as add-on schemes $[7,12]$ for eliminating repetitive disturbances from rotating machinery (such as a disk drive). Because

'Storage Technologies Department, Hewlett-Packard Laboratories, 1501 Page Mill Road, M/S 4U-12, Palo Alto, CA 94304, USA. 2The rule of thumb is that for each drive design the engineer gets to make at most one major change. If a new method requires many changes in order to get any results, it is unlikely to be tried. an adaptive feedforward controller could be added in to an existing design method, it was relatively easy for such a scheme to be adopted into storage systems. Today, adaptive feedforward cancellation of the repetitive runout is a fairly standard feature of disk drive designs. The success of creating a drop-in addition to current control designs has motivated this author's research to follow those lines [1].

Another major iśsue was stated quite eloquently by $\operatorname{Dr}$ Babatunde Ogunnaike, during his plenary talk at the 1998 American Controls Conference in Philadelphia, PA. Dr Ogunnaike was speaking about the chemical process control industry, but much of his talk could apply to any industrial control setting. $\mathrm{He}$ said that building effective control systems in the chemical process industry was $90 \%$ process understanding and 10\% control design. These ratios are certainly true in the data storage industry. While modern control methods put much effort into the creation of nice algorithms for control design, industrial control problems are dominated by an understanding of the particular physical problem. Achieving a good understanding of the particular control problem is justifiably where most of the effort is spent. Thus, a new design methodology which requires substantially more time to understand and implement stands little chance of being used when time is tight. Furthermore, the improvements that can be made by fundamentally understanding the problem and then applying simple solutions typically swamp out the improvements achieved by an improved control design.

An example from this author's work $[2,3,4,6]$ and that of McAllister [8-10] is the generation of air flow by spinning magnetic media. As the disks spin in a disk drive, they carry along air. This in tum generates the air bearing that the magnetic heads 
ride upon. The air flow also generates a process disturbance as it impacts upon the magnetic $\mathrm{read} / \mathrm{write}$ head and the actuator arm. This effect becomes worse as, in fact, spindle speed increases and the air How can become turbulent. This is analogous to an aircraft design problem. A design that is appropriate for an aircraft with a top speed of $300 \mathrm{~km} / \mathrm{h}$ would fail dramatically for an aircraft with a top speed of Mach 2. Likewise, drive designs that were acceptable for lower spindle speeds run into problems tor higher-speed spindles. ${ }^{3}$ The method of improved control design would identify the characteristics of the process disturbance and attempt to design a controller which rejected disturbances in this frequency range. However, by understanding the physical problem it becomes apparent that an aerodynamic redesign of the interior of the disk drive and of the actuator arm achieves such a large reduction in turbulent air flow that conventional control designs can be used. Thus, this is where the effort is spent. Returning to the aircraft design example, there is precious little that one can do with the autopilot on a Boeing 747 to make the plane travel safely at Mach 2.

It is a rather humbling experience but an important lesson to learn. Unfortunately, it is not the only one. As mentioned in the paper, there are severe limitations on the number of calculations that can be done by a control algorithm between sample points. First of all, the sample rate for most hard disk drives is between 4 and $12 \mathrm{kHz}$. For optical disk drives the sample rates can range between 20 and $50 \mathrm{kHz}$. At such high speeds, and given the cost constraints of storage systems, the DSPs that one can afford to put into a disk drive have only a few hundred clock cycles between sample points. One might believe that this still leaves enough operations to calculate some fairly sophisticated control algorithms, but a further restriction is present in that the DSP spends most of its time doing something other than the control law calculation. A number quoted to this author from an engineer at HewlettPackard's former Disk Memory Division was that the DSP spends $95 \%$ of its time doing something other than the control law calculation. Such nonglamorous tasks as calibration, normalisation of inputs, presaturation of outputs, etc. consume most of the code space and clock cycles. For a new, sophisticated algorithm to be used in a disk drive requires that it either be compact enough to replace

\footnotetext{
"The increase in spindle speed is driven by customer desires for faster access times to support such new applications as streaming video from a disk drive. Thus, there is no avoiding the issue.
}

the simple control law or provide such a large improvement in performance to justify a more expensive DSP. When stated this way, the problem of using modern control design methodologies becomes more than a technical issue.

Another issue in the use of modern tools is that of modelling. Most design tools require explicit models of the system - often in state space form, while most drive models are based on instrument measurements of time and frequency responses. The issue then becomes one of transferring the instrument measurement into the modelling package in a form that is usable by the modern tools. This translation is not trivial and requires a different set of low-level programming functions for each new instrument. This again requires extra effort, especially when compared to using a classical control design directly from a Bode plot measured off of a drive.

One final issue not mentioned in the paper was the role that lack of communication plays in limiting the use of modern control methodologies. As mentioned earlier, a tremendous performance improvement can be achieved by understanding the underlying system and how it affects the control system. The converse is also true. Understanding of control systems can point to the key areas where the system design must be improved. Thus, the actual control design problem should be an iterative process involving not only the control engineers, but the mechanical engineers, the material scientists and the device physicists as well. In other words, good industrial control design cannot be separated from good system design and good system design requires a lot of interaction between a lot of different disciplines. When this interaction happens, performance improvements happen largely because a problem which may be hard to fix in one domain is easily eliminated in another domain. Once again this is the issue of process understanding dominating the control design. Unfortunately, the reality of the many industrial situations is that this cross-disciplinary communication is far too rare. Thus, engineers in one area finish their part of the problem and 'throw it over the wall' to the next team. This leaves precious little time for a new control scheme.

All this is not to say that there is no place for modern control techniques in storage system control problems. Over time, many modern control techniques are slowly creeping into the designs. However, they come in one piece at a time and only when conventional methods are deemed inadequate because that is the only way to justify the effort to a budget- and time-conscious manager. This author 
and many others in the field would laud anyone who could show us how to easily "drop in' modern control design into all of our control systems for storage devices.

\section{References}

1. Abramovitch D. Customizable coherent servo demodulation for disk drives. IEEE/ASME Trans Mechatronics 1998; 3: 184-193

2. Abramovitch D, Hurst T, Henze D. The PES Pareto Method: uncovering the strata of position error signals in disk drives. In Proceedings of the 1997 American control conference, Albuquerque, NM. AACC, IEEE. June 1997, pp 2888-2895

3. Abramovitch D, Hurst $T$. Henze D. Decomposition of baseline noise sources in hard disk position error signals using the PES Pareto Method. In: Proceedings of the 1997 American control conference, Albuquerque, NM. AACC. IEEE. June 1997, pp 2901-2905

4. Abramovitch $D$, Hurst $T$, Henze $D$. An overview of the PES Pareto Method for decomposing baseline noise sources in hard disk position error signals. In Digests of the magnetic recording conference 1997 . Minneapolis, MN. IEEE Magnetics Society, IEEE. September 1997

5. Bodson M, Sacks A. Khosia P. Harmonic generation in adaptive feedforward cancellation schemes. IEEE Trans Autom Control 1994; 39 (September)

6. Hurst T, Abramovitch D, Henze D. Measurements for the PES Pareto Method of identifying contributors to disk drive servo system errors. In: Proceedings of the 1997 American control conference, Albuquerque, NM. AACC, IEEE. June 1997, pp 2896-2900

7. Kempf C. Messner W, Tomizuka M. Horowitz R. Comparison of four discrete-time repetitive control algorithms. IEEE Control Syst Mag 1993; 13: 48-54

8. McAllister JS. The effect of disk platter resonances on track misregistration in 3.5 inch disk drives. IEEE Trans Magnetics 1996: 32: 1762-1766

9. McAllister JS. Characterization of disk vibrations on aluminium and altemate substrates. IEEE Trans Magnetics 1996; 33: 968

10. McAllister JS. Disk fiutter: Causes and potential cures. Data Storage 1997; 4: 29-34

11. Sacks A, Bodson M, Messner W. Advanced methods for repeatable runout compensation (disc drives). IEEE Trans Magnetics 1995; 31(2): 1031-1036

12. Tung $E$, Anwar $G$, Tomizuka $M$. Low velocity friction compensation and feedforward solution based on repetitive control. In: Proceedings of the 1991 American control conference. Boston. AACC, IEEE. June 1991. pp 2615-2620

\section{Discussion by Takashi Yamaguchi ${ }^{4}$}

\subsection{Disturbance-Based Control Design Cycle with Experiments Within the Iterative Cycle}

This proposed design method is very impressive from the following viewpoints:

42nd Department, Mechanical Engineering Research laboratory. Hitachi Lld, 502 Kandatsu. Tsuchiura. Ibaraki. 300, Japan.
1. The performance index is the position error signal (PES) itself which is directly one of the drive specifications. The iteration is then included in the design procedure in order to reduce this PES.

2. Concerning this iteration, it seems that conventional $\mathrm{H}$-infinity theory does not give us a methodology to define weighting functions such as $\mathrm{Wt}$ and Ws. There is a guide to define Wt (the weighting function for robust stability), because Wt should be defined by considering the upper bound of model error or variation. But it seems difficult to find a reasonable guide to determine Ws (the weighting function for performance). The proposed method is one solution to determine Wt and Ws.

3. It is very important for this method to have experiments with plural products. In this paper, two drives are used. In actual $R \& D$ activity in industry, it seems difficult to determine how many products for experiment are necessary to design a controller.

\subsection{Seek-Settling Problem}

In the controller of both optical and magnetic disk drives, there are a track-seeking mode (coarse control) and a track-following mode (fine control). One of the big problems is to design seek-settling, in other words, to shape a transient response. The track-following servo controller should have responsibilities for robust stability and disturbance suppression capability, which are the main focusing subjects of this paper. Concerning seek-settling, basically a two-degrees-of-freedom control method is quite effective, as this paper also mentioned. One of the interesting ideas is that the state variables of a track-following mode controller can be replaced by new values at a certain condition. Since the transient response is influenced by the variables, of course, the transient response can be shaped by inputting appropriate values in controller state variables at the beginning of seek-settling. The sudden change of state variable affects transient response very much, but does not change the robust stability or disturbance suppression capability. I suppose this idea would complement the closed loop design shown in this paper [1].

\subsection{Profit/Cost Balance (Section 5.1)}

It is very impressive to point out the "profit/cost balance' of this design. According to my experience of designing $\mathrm{H}$-infinity to hard disk drives, I concur 
with the authors opinion. However, it would be useful to make models of model error and disturbances as a quantitative function for the following reason:

Control engineers have tended to design controllers for a given plant. But, of course a final target of the design is to satisfy product specifications or market requirements. From this viewpoint it is an important activity for control engineers to propose desirable specifications of the plant and disturbances to mechanical engineers. To do so, it is necessary to make clear the specifications of model error and disturbances.

\subsection{Modelling (Section 5.2)}

The three remaining open questions about modelling are shown in Section 5.2. These are very important but very difficult to answer. I suppose that it would be necessary to construct models, considering precise plant characteristics such as tolerances. If a designer who makes models knows some details of the plant, he could understand what uncertainties should be relevant, and which parameter variations would affect plant uncertainty.

\subsection{Performance (Section 5.2)}

Concerning the improvement of closed loop performance, there is a limitation in the area of SISO and linear control. In storage devices, the multi-rate control method has been applied recently. This type of advanced control should be developed.

\subsection{Theory (Section 5.2)}

I concur with the author's opinion. It is important to have a unified design approach including discreteevent control such as a sequence control, and continuous-event control such as a feedback control. For example, it might be necessary to have a switching function to select an appropriate feedback con- troller according to a change of control condition, environment, and injection of disturbances. I suppose a single controller cannot maintain good performance under various conditions, so it seems inevitable that a total control system including discrete-event control will be built in the future. I really agree that there is a lot of work to do.

\section{References}

1. Yamaguchi $T$. et al. A mode-switching controller with initial value compensation for hard disk drive servo control. Control Eng Pract 1997; 5(11): 1525-1532

\section{Final Comments by the Author, M. Steinbuch}

Let me first thank both Dr Yamaguchi, Hitachi, and Dr Abramovich, Hewlett-Packard Labs, for their contribution to the discussion. Both comments contain important additional viewpoints on the issues raised in the paper. In particular I would like to mention the non-triviality of the design of the weighting filters and the relevance of switching control structures in cases of disk drives. Also the point raised with respect to the required systems design approach, i.e. to make organisations such that many disciplines work together instead of local suboptimisation, is very important. *

The original presentation of the paper was at the 'Industry Day' of the European Control Conference, Brussels, 1997. Its title was 'Industrial Feedback', by which I wanted (i) to express how control design is being done in an industrial environment, in addition to showing the implications of using advanced control methods, and (ii) to give feedback from industry to academia about experiences and needs. With this in mind I regret that there is no contribution in the discussion from the academic scene. Do you all agree with the points made in the paper? If so, I expect focus of research and education will change (slowly, I know!) in due time. If you do not agree, I still hope for the same! 